\title{
Article
}

\section{Coverage-dependent structural phase transformations in the adsorption of pentacene on an aperiodically modulated Cu film}

Lahti, M., Pussi, K., Smerdon, Joe, Young, K. M., Sharma, H. R. and McGrath, R.

Available at https://clok.uclan.ac.uk/16802/

Lahti, M., Pussi, K., Smerdon, Joe orcid iconORCID: 0000-0002-7387-8362, Young, K. M., Sharma, H. R. and McGrath, R. (2016) Coverage-dependent structural phase transformations in the adsorption of pentacene on an aperiodically modulated Cu film. The Journal of Chemical Physics, 145 (15). p. 154707. ISSN 0021-9606

It is advisable to refer to the publisher's version if you intend to cite from the work. http://dx.doi.org/10.1063/1.4964920

For more information about UCLan's research in this area go to http://www.uclan.ac.uk/researchgroups/ and search for <name of research Group>.

For information about Research generally at UCLan please go to http://www.uclan.ac.uk/research/

All outputs in CLoK are protected by Intellectual Property Rights law, including Copyright law. Copyright, IPR and Moral Rights for the works on this site are retained by the individual authors and/or other copyright owners. Terms and conditions for use of this material are defined in the policies page. 
Coverage-dependent structural phase transformations in the adsorption of pentacene on an aperiodically modulated $\mathrm{Cu}$ film

M. Lahti, K. Pussi, J. A. Smerdon, K. M. Young, H. R. Sharma, and R. McGrath

Citation: J. Chem. Phys. 145, 154707 (2016); doi: 10.1063/1.4964920

View online: http://dx.doi.org/10.1063/1.4964920

View Table of Contents: http://aip.scitation.org/toc/jcp/145/15

Published by the American Institute of Physics 


\title{
Coverage-dependent structural phase transformations in the adsorption of pentacene on an aperiodically modulated $\mathrm{Cu}$ film
}

\author{
M. Lahti, ${ }^{1}$ K. Pussi, ${ }^{1}$ J. A. Smerdon, ${ }^{2}$ K. M. Young, ${ }^{3}$ H. R. Sharma,${ }^{3}$ and R. McGrath ${ }^{3}$ \\ ${ }^{1}$ School of Engineering Science, Lappeenranta University of Technology, P.O. Box 20, \\ FIN-53851 Lappeenranta, Finland \\ ${ }_{2}^{2}$ Jeremiah Horrocks Institute for Mathematics, Physics and Astronomy, University of Central Lancashire, \\ Preston PR1 2HE, United Kingdom \\ ${ }^{3}$ Surface Science Research Centre, University of Liverpool, Oxford St., Liverpool L69 3BX, United Kingdom
}

(Received 11 May 2016; accepted 3 October 2016; published online 21 October 2016)

\begin{abstract}
Surface ordering of pentacene molecules adsorbed on an aperiodic $\mathrm{Cu}$ surface has been studied with density functional theory (DFT) and scanning tunnelling microscopy as a function of coverage. Below $0.73 \mathrm{ML}\left(5.3 \times 10^{13}\right.$ molecules $\left.\mathrm{cm}^{-2}\right)$, the adsorbate structure is row-like with the molecular axes aligned with the rows in the $\mathrm{Cu}$ structure. Between this coverage and $1 \mathrm{ML}\left(7.3 \times 10^{13}\right.$ molecules $\mathrm{cm}^{-2}$ ), a structural phase with a checkerboard structure is seen. At this coverage region, the molecules are very close to each other which leads to unusual bending. At higher coverages, a further phase transition to a high-density row structure is seen for most of the film. DFT with van der Waals functionals is employed to study how the molecule-molecule and molecule-surface interactions evolve as a function of coverage. Published by AIP Publishing. [http://dx.doi.org/10.1063/1.4964920]
\end{abstract}

\section{INTRODUCTION}

The study of aperiodic structures and their properties is an area of surface science which continues to evoke interest and attention. ${ }^{1,2}$ The interplay of complex aperiodic surface structure and properties (including adsorption) and the relation to electronic structure are relatively unexplored and yet offer a rich testing ground for fundamental ideas of order and symmetry and their influence on physical systems.

Vicinal (highly stepped, one or more large Miller indices) surfaces have been the focus of some attention in the past decade for their promise in inducing one-dimensional arrangement of adsorbates. However, they are very disordered due to low coordination.

A thin film of $\mathrm{Cu}$ on the fivefold surface of Al-Pd-Mn forms a stable, well-ordered structure that is uniaxially commensurate with the aperiodic structure of the substrate. ${ }^{3}$ This structure has been analysed using low-energy electron diffraction and is found to consist of a vicinal surface of a body-centered tetragonal (bct) (100) structure. This bct(100) structure has lattice parameters of $\mathrm{a}=2.88 \AA, \mathrm{b}=2.55 \AA$ and $\mathrm{c}=2.88 \AA$, with the vicinal surface making an angle $\alpha$ of $13.28^{\circ}$ relative to the $\mathrm{a}-\mathrm{b}$ plane. ${ }^{4}$ This results in a surface with a very dense pattern of steps which is better ordered than any conventional vicinal surface such as $\mathrm{Cu}(119)$; therefore this system provides an ideal opportunity to explore the effects of adsorption on a vicinal aperiodic surface.

The use of density functional theory (DFT) in combination with advanced experimental techniques has facilitated the investigation of such increasingly complex surface systems. In this study, we build on previous results for molecular adsorption on an aperiodically modulated $\mathrm{Cu}$ thin film. We use an improved DFT methodology to quantify previously unresolved parameters of the adsorption system and show that this new information allows a simple explanation of the coverage-dependent structural phase transitions observed in the adsorbed molecular overlayer.

Pentacene $\left(\mathrm{C}_{22} \mathrm{H}_{14}\right.$, dimensions, $\left.1.42 \times 0.5 \mathrm{~nm}, \mathrm{Pn}\right)$ is an ambipolar organic semiconductor consisting of five linearly bonded benzene rings. It is used as a $p$-type molecule for organic field effect transistors and it has received much attention because of its unusually high intrinsic charge carrier mobility without doping. Significant deviation from a conventional planar adsorption configuration has been reported for $\mathrm{Au}(110)$, with mixed edge on/planar phases ${ }^{5}$ and for $\mathrm{Al}(001)$, with a peculiar V-shaped bending. ${ }^{6}$ Otherwise, a minor symmetrical bending of the molecule with the central ring closer to the surface has been reported for adsorption on $\mathrm{Au}(111),{ }^{7} \mathrm{Cu}(001),{ }^{8} \mathrm{Cu}(111),{ }^{9}$ and $\mathrm{Cu}(110){ }^{10}$ surfaces. The adsorption site on $\mathrm{Cu}(111)$ at low temperature was revealed by Lagoute et al. ${ }^{11}$ using scanning tunnelling microscopy (STM). Its interactions with $\mathrm{Ag}(111)$ and $\mathrm{Cu}(111)$ have also been investigated with $\mathrm{x}$-ray standing waves to determine the adsorption height. ${ }^{12}$ It was found that the arrangement of surface steps could influence the orientation of bulk films grown on $\mathrm{Si}(111) .^{13}$ In the effort to grow thin films of aligned $\mathrm{Pn}$ molecules, the use of $\mathrm{Cu}(119)$ as a vicinal substrate was explored using low-energy electron diffraction, ultraviolet photoelectron spectroscopy, ${ }^{14}$ and scanning tunneling microscopy (STM). ${ }^{15}$

In our previous article, ${ }^{16}$ we addressed the trends for Pn adsorption by studying the adsorption of anthracene and naphthalene on this aperiodic $\mathrm{Cu}$ surface with density functional theory (DFT). Because of limited computational resources, we did not use van der Waals (vdW) functionals. In this article, we study both the ordering as a function of coverage and bending of a Pn molecule adsorbed on the 
aforementioned aperiodic copper surface with DFT and STM. DFT with vdW functionals is employed to study how the molecule-molecule and molecule-surface interactions evolve as a function of coverage.

\section{EXPERIMENTAL DETAILS}

The sample was grown at the Ames Laboratory with a nominal composition of $\mathrm{Al}_{70} \mathrm{Pd}_{21} \mathrm{Mn}_{9}$ and cut perpendicular to a fivefold axis. ${ }^{17} \mathrm{Pn}$ was evaporated from a Pyrex tube wrapped with a $\mathrm{W}$ filament and a thermocouple for temperature regulation and $\mathrm{Cu}$ was evaporated from a simple filament source consisting of a piece of OFHC Cu wrapped with a W filament. All depositions and data collection were carried out with the sample maintained at room temperature. A variabletemperature Omicron STM was used for the collection of STM data. The experimental data presented are collected as detailed earlier. ${ }^{16}$

\section{A. Coverage definition}

The use of monolayers (MLs) as a unit of coverage is intuitive, though not very transparent when there are densityrelated phase transitions in a molecular overlayer. For this reason, our earlier report uses the unit of molecules $\mathrm{cm}^{-2} .{ }^{16}$ However, the definition of $1 \mathrm{ML}$ as the coverage at which the number of $\mathrm{C}$ atoms in adsorbed Pn molecules is the same as the number of surface $\mathrm{Cu}$ atoms is intuitive and convenient for describing DFT results and is also the coverage at which the checkerboard structure observed previously ${ }^{16}$ and examined here is saturated. To take advantage of both schemes, we present coverages side-by-side in both ML and molecules $\mathrm{cm}^{-2}$, with $1 \mathrm{ML}$ equal to $7.3 \times 10^{13}$ molecules $\mathrm{cm}^{-2}$.

\section{COMPUTATIONAL DETAILS}

The static calculations for total energies were performed using the Vienna $a b$ initio simulation package (VASP) ${ }^{18-22}$ including the projector augmented wave (PAW) ${ }^{23}$ potentials. A kinetic energy cutoff of $400 \mathrm{eV}$ was applied for the plane waves. The exchange and correlation functionals were treated by the generalized-gradient approximation (GGA) of Perdew-Burke-Ernzerhof (PBE) as proposed by Perdew, Burke, and Ernzerhof. ${ }^{24}$ The $8 \times 8 \times 1$ Monkhorst-Pack $\operatorname{mesh}^{25}$ was used for $\mathrm{k}$-point sampling. The $\mathrm{Cu}$ surface was modelled using the supercell approach, where periodic boundary conditions are applied to the central supercell. The surface slab was modelled with 5 layers of $\mathrm{Cu}$. The number of $\mathrm{Cu}$ atoms was between 138 atoms and 299 atoms depending on the coverage and the surface structure. A region of approximately $20 \AA$ of vacuum was inserted in the $z$-direction to prevent interactions occurring between periodic images. The bottom layer of the surface slab was fixed during geometry relaxation. For the description of longrange vdW energy, the optB86b-vdW functional ${ }^{26}$ was used. This functional gives the experimental lattice constant for $\mathrm{Cu}$ and also gives good agreement for the adsorption energy of benzene on $\mathrm{Cu}$ surfaces. ${ }^{27,28}$
The adsorption energies of molecules are defined as

$$
E_{a d s}=\frac{1}{N}\left(E_{\text {tot }}-E_{\text {clean }}-N E_{m o l}\right),
$$

where $E_{\text {tot }}$ is the total energy of a relaxed supercell with molecules, $\mathrm{E}_{\text {clean }}$ is the total energy of the relaxed clean $\mathrm{Cu}$ slab, $\mathrm{N}$ is the number of molecules, and $\mathrm{E}_{\mathrm{mol}}$ is the energy of one molecule in the space.

In the calculation of Pn adsorption sites, two different supercells are used: one with 138 atoms and the other with 184 atoms. The number of surface $\mathrm{Cu}$ atoms was 30 and 40 , respectively. The smaller supercell was mainly used to reduce the level of computational resources required. From the starting configuration, full relaxation of the atomic positions was allowed until the forces were minimized. In the relaxed configuration, the molecule lies flat on the surface.

\section{RESULTS}

\section{A. Clean Fibonacci modulated copper surface}

The Fibonacci modulated $\mathrm{Cu}$ surface contains two different kinds of fcc(100) oriented terraces: long (L) and short (S), separated by fcc(111) oriented steps and arranged in a binary Fibonacci sequence (LSLLS...). The film grows in layers parallel to the substrate and contains inter-layer steps; henceforth we differentiate the intra-layer fcc(111) oriented steps by referring to them as Fibonacci rows and to the overall structure as the Fibonacci row structure. Long terraces are three atomic rows wide and short terraces are two atomic rows wide. The density of surface $\mathrm{Cu}$ atoms is around $63 \%$ of that of a $\mathrm{Cu}(100)$ surface, though it is not as well defined for this complicated surface. The Fibonacci row structure is very dense $(\mathrm{L}=7.3 \AA, \mathrm{S}=4.5 \AA$ ), therefore, each adjacent row cannot be decorated by an unbroken chain of molecules. ${ }^{16}$ This surface is therefore unique in at least two regards: first, it is aperiodic, in the sense that a periodic structure is perturbed by an aperiodic modulation; second, it is vicinal, in the sense that there is a well-defined cut plane across an underlying periodic structure. As it is also flat and well ordered, it differs from other vicinal surfaces which are usually quite disordered due to low coordination.

The structure is inherently three-dimensional, exhibiting lesser coverage with each additional layer, resulting in a sparse top layer of $\mathrm{Cu}$ islands atop at least one more layer with gaps, in turn atop at least one continuous layer of $\mathrm{Cu}$ covering the substrate. Despite this, the film is predominantly flat, with much more of the $\mathrm{Cu}$ surface parallel to the substrate surface than is occupied in forming interlayer steps. We focus on adsorption on the "flat" $\mathrm{Cu}$ surface and neglect stepwise adsorption, which is likely to show the same tendency as for any other stepped surface.

The relaxation of this $\mathrm{Cu}$ surface was previously studied using DFT without taking into account the vdW interactions. These calculations indicated that the symmetry of the clean surface remained almost constant, though the edge row of the longer terrace falls slightly. ${ }^{16}$ In the case of calculations with vdW functionals, surface distortion occurs during the relaxation of the clean surface. The lattice constant of $\mathrm{Cu}$ 
found with the optB86b-vdW functional is convergent with the experimental lattice constant which makes this result more reliable than in the case of calculations without $\mathrm{vdW}$ functionals. ${ }^{28}$ In the distortion, the edge row of the longer terrace moves to a new position and causes modifications to the whole surface. However, the largest change is a gap between the second and third row of the longer terrace, which makes the $\mathrm{L}$ terraces locally wider (resembling $\mathrm{Cu}(110)$ ) and probably increases the reactivity at this local site. The distance between the second and the third atomic row on $\mathrm{L}$ terraces before and after surface distortion is $2.9 \AA$ and $3.6 \AA$, illustrated in Figure 1.

\section{B. Pn adsorption}

\section{Scanning tunneling microscopy}

In Figure 2, we present STM data collected from this system at various coverages. The islands observed in the STM topographs are $\mathrm{Cu}$ islands due to the three-dimensional nature of the $\mathrm{Cu}$ growth mode, rather than the islands of pentacene molecules, which are observed individually as rods of approximately $2 \mathrm{~nm} \times 0.4 \mathrm{~nm}$. Coverages are obtained by counting molecules and are approximate due to the nonuniformity of the coverage and the occasional lack of definition in the molecules. The largest source of error is the indistinctness of the molecules, which we estimate leads to an error of approximately $10 \%$. Counting is done on STM data where the molecules are resolved, i.e., when some of the surface is covered with the checkerboard structure, which presents a comparison both for the uncovered surface and for the surface covered by the row structure, which are for the most part indistinguishable otherwise. Coverages are estimated based on deposition time for images without the visible checkerboard structure. Due to the patches of $\mathrm{Cu}$ free of Pn molecules observed in all our data, even if all of the (a)
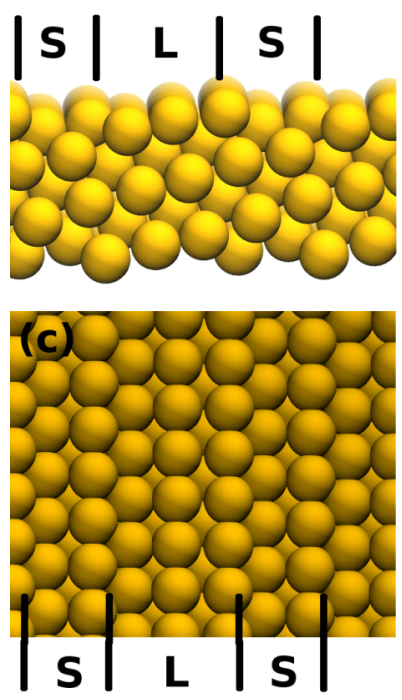

(b)
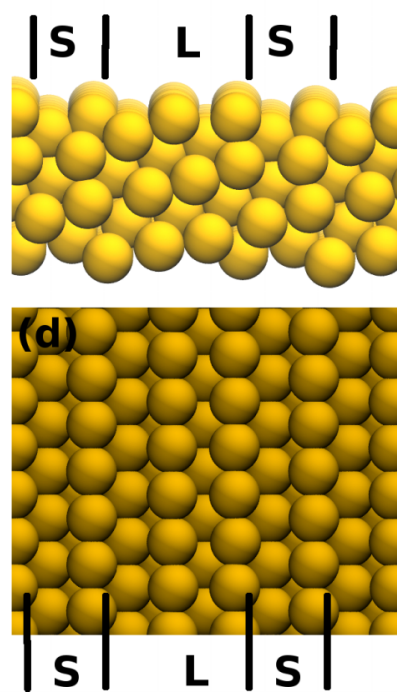

FIG. 1. Clean Fibonacci modulated Cu surface. (a) Side view of the model surface before relaxation. (c) Top view of the surface before relaxation. (b) Side view of the distorted surface. (d) Top view of the distorted surface.
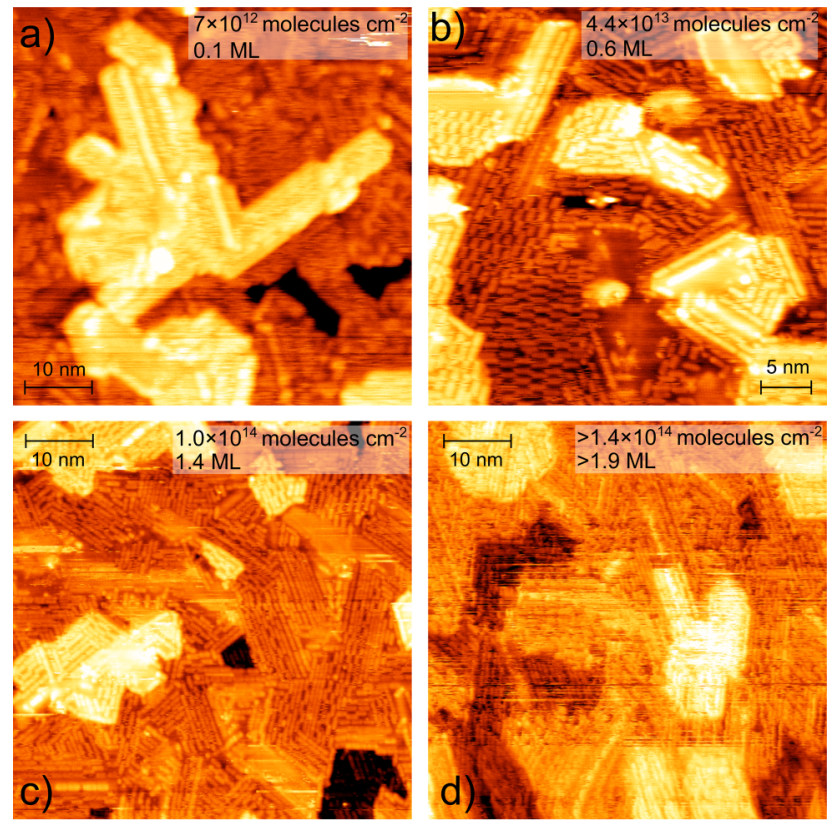

FIG. 2. Room temperature scanning tunneling microscopy of the evolution of the Pn structure atop Fibonacci-modulated $\mathrm{Cu} / \mathrm{AlPdMn}$ with coverage. Quoted coverages are estimated to be accurate within 10\%. Quoted voltages are sample bias. Data are unprocessed except for leveling and a false color map. (a) $60 \mathrm{~nm} \times 60 \mathrm{~nm}, \mathrm{I}_{\mathrm{T}}=0.11 \mathrm{nA}, \mathrm{V}_{\mathrm{B}}=1.03 \mathrm{~V}$. The linear features are end-to-end Pn rows. Between some of these rows are parallel rows with significant noise, which indicates there are mobile pentacene molecules on the surface. (b) $\mathrm{I}_{\mathrm{T}}=0.087 \mathrm{nA}, \mathrm{V}_{\mathrm{B}}=-1.14 \mathrm{~V}$. The checkerboard pattern is clearly visible. Noise is greatly reduced. Another STM image of this area is published in Ref. 16. (c) $\mathrm{I}_{\mathrm{T}}=0.1 \mathrm{nA}, \mathrm{V}_{\mathrm{B}}=-1 \mathrm{~V}$. The checkerboard pattern no longer dominates and more Pn molecules are lined up in a row structure. (d) $\mathrm{I}_{\mathrm{T}}=0.1 \mathrm{nA}, \mathrm{V}_{\mathrm{B}}=-1.3 \mathrm{~V}$. No checkerboard structure is visible. The data are very noisy due to the diffusion of loosely bound second layer molecules.

Pn molecules are in the checkerboard structure, the coverage is less than the maximum checkerboard coverage of $0.98 \mathrm{ML}$ or $7.1 \cdot 10^{13}$ molecules $\mathrm{cm}^{-2}$.

The noise evident in the topographs is due to loosely bound molecules moving through some combination of thermal diffusion and interaction with the STM tip. This noise is particularly evident in Panel (a), where coverage is very low. Several features are apparently noise-free, notably steps in the $\mathrm{Cu}$ film and long end-to-end rows of Pn molecules, which are locations where $\mathrm{Pn}$ motion is restricted due to increased coordination with $\mathrm{Cu}$ or with coadsorbates. In Panel (b), less noise is evident, as most of the available sites for Pn adsorption are occupied, which has the effect of "locking in" the structure. Some portions of the aperiodic $\mathrm{Cu}$ substrate are completely clear of Pn molecules, for reasons which are not apparent. Nearly all of the molecular film, however, is in the checkerboard structure described previously. ${ }^{16}$ As coverage increases between Panels (b) and (c), we see a reduction in the proportion of material manifesting the checkerboard structure.

Since we have no reason to expect that material has desorbed, we find it reasonable to extrapolate that the lack of checkerboard material observed in Panel (c) indicates a transformation to some other structure. The STM data point to a return to the row structure but with a tighter inter-row packing. Certain areas appear free of Pn molecules but as they 
appear to be at the height of the neighboring Pn molecules, we suggest that they are in fact closely packed Pn molecules which are not resolved. There is very little noise in this image, supporting the idea that with increasing coverage the mobility of Pn molecules is restricted. What noise is observed is higher in $z$ than adjacent data, suggesting that it is due to the nascent second layer. In Panel (d), coverage is too high to accommodate all molecules in the first layer, as indicated by the large degree of noise originating in the diffusion of second-layer molecules.

If we consider a two-dimensional model system consisting of rigid, round-ended, finite width, finite breadth, elongated three-dimensional batons aligned along their long axes on a set of parallel lines with a separation comparable to the baton dimensions, a checkerboard packing provides both the maximum density and the maximum nearest-neighbour distance of batons. This is sufficient reasoning to understand the adoption of the checkerboard structure, given the attraction to the substrate and the close-range intermolecular repulsion of the Pn molecules, regardless of any additional substratemediated repulsion found in previous work. ${ }^{16}$

In Figure 3, we focus on the second phase transition: the transition from checkerboard to another row structure. However, it is clear that the row structure following the second phase transition contains a higher density of Pn molecules than that preceding the first phase transition. In addition, as highlighted in the center of Panel (b), not all rows are of the same height as image via STM. In the lower right portion of Panel (b), the black and white dashes indicate a region where a row structure of $4 \mathrm{dim}$ transitions to a structure of $3 \mathrm{dim}$ and 2 bright in the same cross-rows distance. These pieces of information indicate the freedom of the molecules to tilt into the third dimension and thus reduce their surface footprint. This process enables molecules to adsorb on more Fibonacci rows and thus to increase the film density. This is the second phase transition.
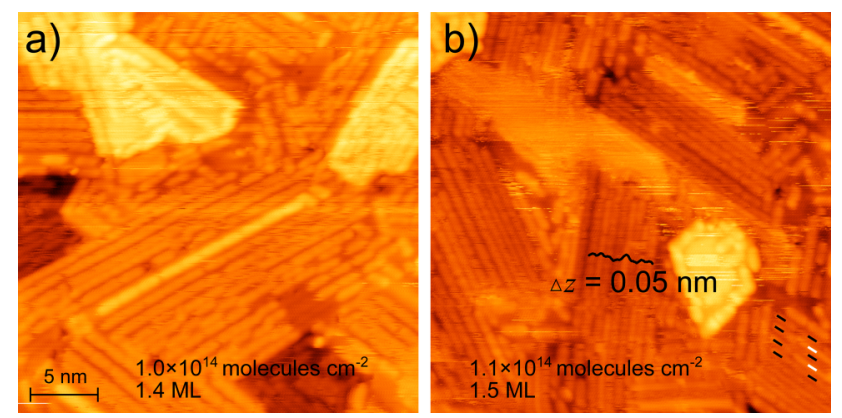

FIG. 3. $30 \mathrm{~nm} \times 30 \mathrm{~nm}$ topographs of the $\mathrm{Pn} / \mathrm{Cu} / \mathrm{AlPdMn}$ surface. (a) $\mathrm{I}_{\mathrm{T}}=150 \mathrm{pA}, \mathrm{V}_{\mathrm{B}}=1.6 \mathrm{~V}$. Checkerboard structure has been almost completely replaced by another row structure. Noise is observed around some molecules in the remaining checkerboard structure, indicating that these molecules have become unstable in this adsorption configuration. (b) $\mathrm{I}_{\mathrm{T}}$ $=110 \mathrm{pA}, \mathrm{V}_{\mathrm{B}}=1.05 \mathrm{~V}$. Slightly higher coverage. No checkerboard Pn remains. The differing heights of the Pn rows shown in the profile are likely to be evidence of tilted rows as explained in the text. $\Delta \mathrm{z}$ given is between the tops of adjacent rows and does not include the Pn layer thickness. In the lower right portion of the image, a transition between 4 flat rows and 3 flat +2 tilted rows in the same cross-rows distance is indicated. An additional example is shown in the upper middle portion, where 3 flat rows have a small section with an additional tilted row inserted.
The second phase transition seems to occur with relatively little disruption to the film. The first phase transition involves a large-scale reorganization. We expect that this reorganization energy cost is absorbed by the thermal energy which the molecules already possess, as can be seen from the ready diffusion of the molecules at this temperature.

\section{Density functional theory with van der Waals}

a. Pn adsorption sites. The sites considered for Pn adsorption were selected based on our previous study ${ }^{16}$ and the geometry of the surface. In all cases, adsorption crosswise to the Fibonacci rows was less favorable than adsorption parallel to the rows. We include the results for crosswise adsorption in the supplementary material.

The sites for parallel adsorption are shown in Figure 4 and the associated adsorption energies in Table I. A larger negative energy indicates a stronger attraction. The best adsorption sites on the smaller supercell (1 and 5) were also tested with the larger supercell, along with adsorption site 4 in order to give another reference point. The adsorption sites in the figures are plotted before and after relaxation except for the sites 8,9 , and 10, which are missing the after geometry as the molecules on these sites drifted away from their before geometries. Pn molecules starting from sites 9 and 10 drifted to site number 1 , and those from site 8 drifted to site number 5 .

The 3 sites with the highest negative energy of adsorption are 1,11 , and 5.1 and 11 are translated approximately 0.5 $\mathrm{Cu}$ nearest neighbour distance with respect to each other. The similarity in adsorption energy of these two sites indicates a low barrier for diffusion along the Fibonacci rows. The postrelaxation Fibonacci L-row resembles the $\mathrm{Cu}(110)$ surface, and site 11 is chosen as it corresponds to the best adsorption site on the $\mathrm{Cu}(110)$ surface. $^{29}$

b. Surface structures as a function of coverage. As coverage is varied, the superstructure of the Pn layer changes. As the STM data show, this change is not continuous but

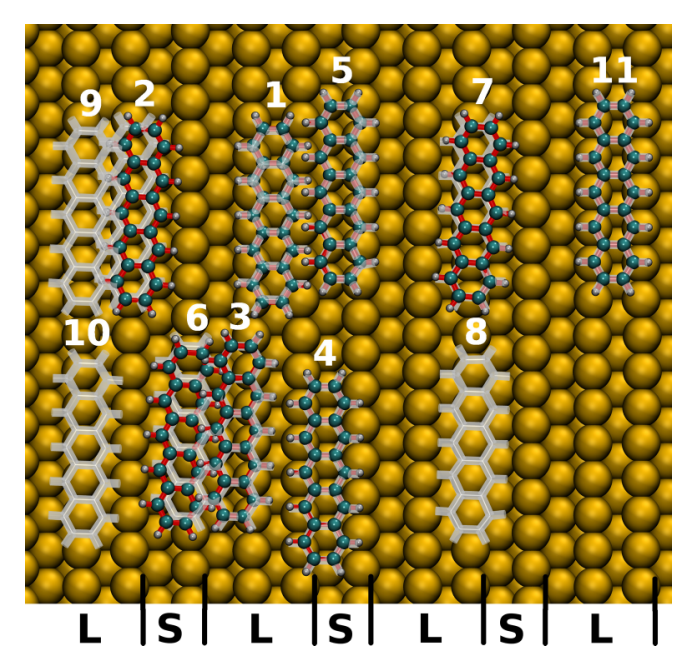

FIG. 4. Pn adsorption on the Fibonacci modulated copper surface. Adsorption sites for Pn adsorption parallel to the steps. Adsorption energies are listed in Table I. 
TABLE I. Adsorption energies for the adsorption sites shown in Figure 4. The two right hand columns refer to the two different sizes of $\mathrm{Cu}$ slab used in the calculation.

\begin{tabular}{lcc}
\hline \hline & Adsorption energy (eV) & Adsorption energy (eV) \\
& $0.733 \mathrm{ML}$ & $0.55 \mathrm{ML}$ \\
Adsorption site & $5.3 \times 10^{13}$ molecules cm $\mathrm{cm}^{-2}$ & $4 \times 10^{13}$ molecules cm $^{-2}$ \\
\hline 1 & -4.36 & -4.72 \\
2 & -3.15 & \\
3 & -3.67 & -3.70 \\
4 & -3.14 & -4.18 \\
5 & -3.73 & \\
6 & -3.29 & \\
7 & -2.83 & \\
8 & Not stable & \\
9 & Not stable & -4.44 \\
10 & Not stable & \\
11 & -4.06 & \\
\hline
\end{tabular}

has three main phases separated by two phase transitions. When the coverage is low enough, all the molecules stick to the energetically most favorable adsorption sites as expected. In this situation, we do not observe via DFT any interaction between the molecules. When the coverage is increased and all of the most favorable sites are occupied, the molecules start to occupy the next available best adsorption sites. Placing the molecules at the simultaneously available two most favorable sites produces the checkerboard structure. The calculated checkerboard structure at a coverage of $0.98 \mathrm{ML}\left(7.1 \times 10^{13}\right.$ molecules $\left.\mathrm{cm}^{-2}\right)$ is shown in Fig. 5.

The first superstructure phase transition occurs as coverage exceeds $0.73 \mathrm{ML}\left(5.3 \times 10^{13}\right.$ molecules $\left.\mathrm{cm}^{-2}\right)$. Below this coverage, the row structure is observed as the molecule can adopt the best adsorption sites. Above

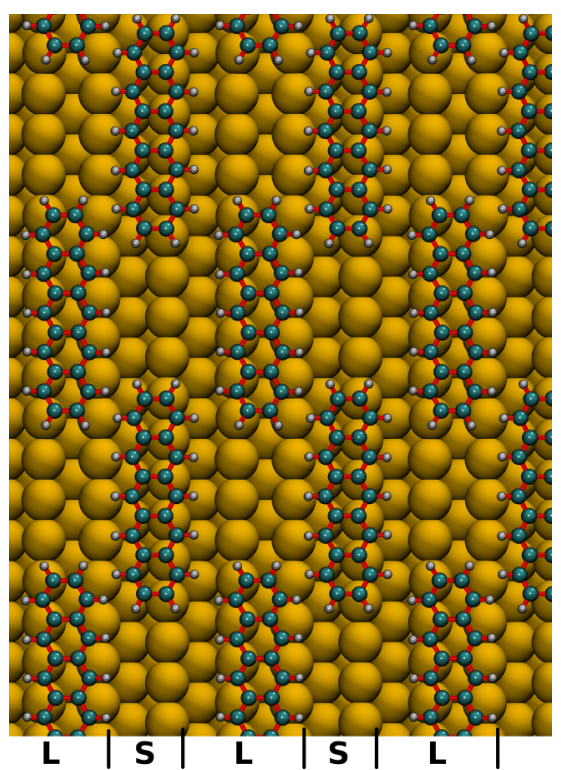

FIG. 5. Pn adsorption on the Fibonacci modulated copper surface. Calculated checkerboard structure with the coverage of $0.98 \mathrm{ML}$ (7.1 $\times 10^{13}$ molecules $\mathrm{cm}^{-2}$ ). this coverage, the checkerboard structure is energetically most favorable. The intermolecular repulsion starts to overwhelm the surface attraction as the coverage increases from $0.55 \mathrm{ML}\left(4 \times 10^{13}\right.$ molecules $\left.\mathrm{cm}^{-2}\right)$ to $0.73 \mathrm{ML}$ $\left(5.3 \times 10^{13}\right.$ molecules $\left.\mathrm{cm}^{-2}\right)$, reflected in the decrease in magnitude of the per-molecule adsorption energy. It is impossible to maintain a flat-lying row structure with coverage above $0.73 \mathrm{ML}\left(5.3 \times 10^{13}\right.$ molecules $\left.\mathrm{cm}^{-2}\right)$ because after that molecules start to overlap with each other.

c. Tilted rows. Raising the number of molecules beyond that which can fit in a checkerboard structure (0.98 ML/7.1 $\times 10^{13}$ molecules $\mathrm{cm}^{-2}$ ) leads to a tilted row structure. This is the second phase transition. DFT simulations are run for the coverage of $1.46 \mathrm{ML}\left(1.1 \times 10^{14}\right.$ molecules $\left.\mathrm{cm}^{-2}\right)$ as shown in Figure 6. This high coverage structure illustrates a situation where all Fibonacci rows are occupied and on each Fibonacci row, the Pn molecules are as close as they can get. The shortest vertical distance between the $\mathrm{C}$ atoms of the Pn molecule and the $\mathrm{Cu}$ atoms of the substrate is $2.2 \AA$ for both $\mathrm{L}$ and S Fibonacci rows. The distance between the Pn molecules measured between the $\mathrm{H}$ atoms is $2.0 \AA$ for the molecules on the same terrace and $2.2 \AA$ for the molecules that are on different terraces. In addition to the bending of the Pn molecules, we also see tilting of about $30^{\circ}$ of the molecules that sit on the S-terrace.

The adsorption energy also varies with the coverage. The most negative per-molecule values are observed for low coverage. For the checkerboard structure at a coverage of $0.73 \mathrm{ML}\left(5.3 \times 10^{13}\right.$ molecules $\left.\mathrm{cm}^{-2}\right)$, the adsorption energy was $-4.40 \mathrm{eV}$. The adsorption energy of $\mathrm{Pn}$ on $\mathrm{Cu}(111)^{9}$ is calculated, using the same method, as $-3.17 \mathrm{eV}$. This indicates that the bonding between the Fibonacci modulated surface and the molecule is stronger than that for a flat surface.

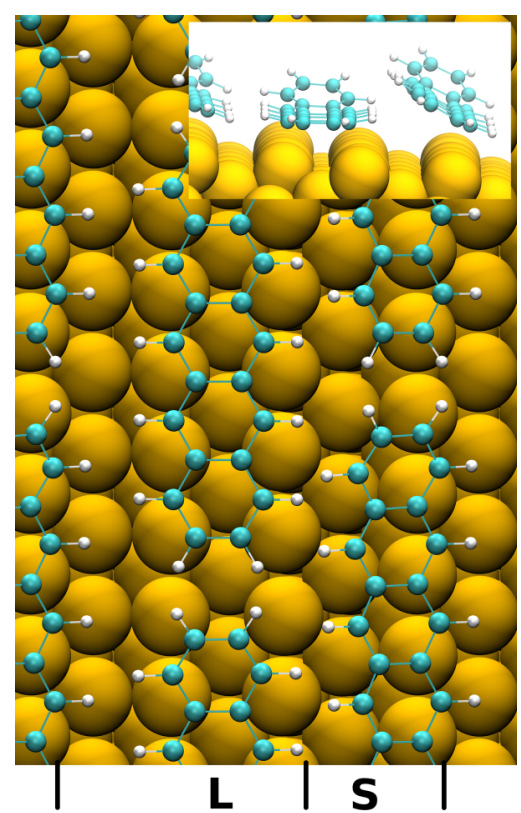

FIG. 6. Pn adsorption on the Fibonacci modulated copper surface. Calculated high coverage structure with the coverage of $1.46 \mathrm{ML}$. The insert shows the tilting of the Pn molecule. 
a)

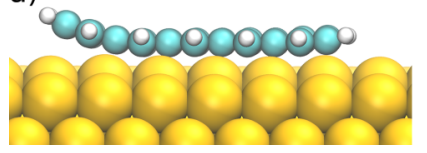

b)

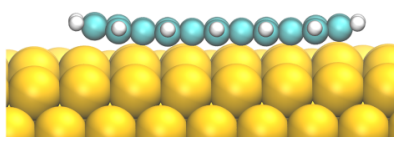

FIG. 7. Pn adsorption on the Fibonacci modulated copper surface. Bending of the Pn molecule at a coverage of (a) $0.733 \mathrm{ML}\left(5.3 \times 10^{13}\right.$ molecules cm $\left.\mathrm{cm}^{-2}\right)$ and (b) $0.40 \mathrm{ML}\left(2.9 \times 10^{13}\right.$ molecules $\left.\mathrm{cm}^{-2}\right)$.

In the case of the Fibonacci modulated $\mathrm{Cu}$ surface, it is hard to unambiguously define the adsorption height, so we measure the smallest $\mathrm{C}-\mathrm{Cu}$ distance, which is $2.1 \AA$ for adsorption site 1 . On the $\mathrm{Cu}(111)$ surface, the distance between the molecule and the surface is experimentally measured to be $2.34 \AA .{ }^{12}$ The shorter adsorption height for our surface also supports the assumption of stronger bonding.

d. Bending of Pn molecule. On low Miller index $\mathrm{Cu}$ planes, the Pn molecule experiences a bending that can clearly be seen with STM $^{8,10,11,30-32}$ and atomic force microscopy (AFM) ${ }^{33}$ The bending of an isolated Pn molecule has also been quantified using DFT. ${ }^{6,8,9,32}$ The bending on the $\mathrm{Cu}(001)$ surface is approximately $0.4 \AA$ measured between the center of the molecule and a plane bisecting the $\mathrm{H}$ atoms at either ends, ${ }^{10}$ and on $\mathrm{Cu}(100)$ and $\mathrm{Cu}(111)$ surfaces, the values are $0.16 \AA$ and $0.2 \AA$ measured between the center of the molecule and a plane bisecting the $\mathrm{C}$ atoms at either ends. ${ }^{8,9} \mathrm{Al}(001)$ shows a larger value for bending with peculiar $\mathrm{V}$-shape, where the height difference between the peripheral and central carbon atoms is $1.24 \AA^{\circ} .^{6}$ The bending of Pn molecules seems to be driven by something in addition to optimizing $\mathrm{C}-\mathrm{Cu}$ distance, as "chain-like" behavior is observed atop $\mathrm{Cu}(111)$, where one end of a molecule in a closely-packed layer is forced beneath the end of a neighboring molecule, causing it to tilt and propagate the effect to the next neighbor. Bending is also observed for molecules in the second layer. $^{32}$

Figure 7 shows the side views of Pn bending at two different coverages. Table II shows parameters derived from DFT related to the bending of the Pn molecule on the Fibonacci modulated copper surface with different coverages (see Figure 8 for a graphical explanation of the different parameters). Bending is measured between the center of

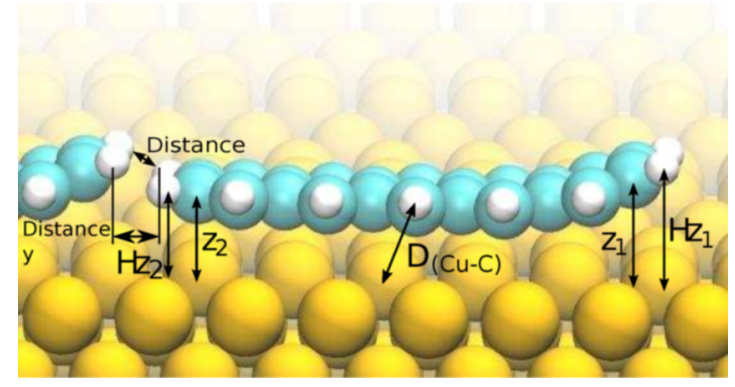

FIG. 8. Pn adsorption on the Fibonacci modulated copper surface. Graphical explanation for the main parameters in Table II.

the molecule and a plane bisecting the $\mathrm{C}$ atoms at either ends of the molecule (surface plane is not unambiguous to define). Table II also shows bending measured between the center of the molecule and a plane bisecting the $\mathrm{H}$ atoms at either ends. Although the bending of Pn molecules can usually be seen via STM, the noise in our images make a measurement intractable. However, in the image in Figure 3(b), the molecules in the rows have slightly increased definition, which could be a manifestation of this bending.

Columns Distance $y$ and Distance in Table II show the distances between the molecules in two different ways. The first one (distance $y$ ) means the lateral distance between $\mathrm{H}$ atoms of the neighbouring molecules. The other (distance) is the true distance between the last $\mathrm{H}$ atom of the molecule and the first $\mathrm{H}$ atom of the next molecule. The only coverage where those two measures are different is $0.733 \mathrm{ML}$ $\left(5.3 \times 10^{13}\right.$ molecules $\left.\mathrm{cm}^{-2}\right)$. At this coverage, there is a strong bending of one end of the molecule that affects the real distance. At this coverage, the molecules are very close to each other and this clearly causes unusual bending. Repulsion between the ends of the molecules forces a chain effect where one end of a molecule bends up and one end of a neighbouring molecule bends down, as observed for $\mathrm{Cu}(111) .{ }^{32}$ This causes an overall decrease in the adsorption energy.

With lower coverages the bending decreases but there is still some variation. Bending seems to be extremely sensitive to changes in the electrical environmentespecially considering that the bending difference be-

TABLE II. Parameters related to the bending of the Pn molecule as a function of coverage. Column $z_{1}$ represents one end of the molecule and column $z_{2}$ represents another end of the molecule. Column $\mathrm{z}_{\mathrm{a}}$ represents the average value of $\mathrm{z}_{1}$ and $\mathrm{z}_{2}$. Columns $\mathrm{Hz}_{1}, \mathrm{~Hz} \mathrm{z}_{2}$, and $\mathrm{Hz} \mathrm{z}_{\mathrm{a}}$ correspond to the columns $\mathrm{z}_{1}, \mathrm{z}_{2}$, and $\mathrm{z}_{\mathrm{a}}$. Column $\mathrm{D}_{(\mathrm{Cu}-\mathrm{C})}$ represents a shortest distance between $\mathrm{C}$ in the molecule and $\mathrm{Cu}$ atom at the surface (bond length). Column $\mathrm{A}_{\mathrm{h}}$ represents the average height of the $\mathrm{C}$ atoms measured from the row of highest atoms of the surface. See Figure 8 for the graphical explanation of the main parameters.

\begin{tabular}{|c|c|c|c|c|c|c|c|c|c|c|}
\hline $\begin{array}{l}\text { Coverage (ML } \\
\left.\left(\cdot 10^{13} \text { molecules } \mathrm{cm}^{-2}\right)\right)\end{array}$ & Distance y $(\AA)$ & Distance $(\AA)$ & $\mathrm{z}_{1}(\AA)$ & $\mathrm{z}_{2}(\AA)$ & $\mathrm{z}_{\mathrm{a}}(\AA)$ & $\mathrm{Hz}_{1}(\AA)$ & $\mathrm{Hz}_{2}(\AA)$ & $\mathrm{Hz}_{\mathrm{a}}(\AA)$ & $\mathrm{D}_{(\mathrm{Cu}-\mathrm{C})}(\AA)$ & $\mathrm{A}_{\mathrm{h}}(\AA)$ \\
\hline $0.34(2.4)$ & 19.15 & 19.15 & 0.32 & 0.18 & 0.25 & 0.58 & 0.45 & 0.52 & 2.203 & 2.077 \\
\hline 0.37 (2.7) & 16.59 & 16.59 & 0.34 & 0.23 & 0.29 & 0.57 & 0.47 & 0.52 & 2.209 & 2.081 \\
\hline $0.40(2.9)$ & 14.04 & 14.04 & 0.35 & 0.21 & 0.28 & 0.55 & 0.44 & 0.50 & 2.228 & 2.061 \\
\hline $0.44(3.2)$ & 11.43 & 11.43 & 0.36 & 0.21 & 0.29 & 0.53 & 0.40 & 0.47 & 2.225 & 2.053 \\
\hline $0.49(3.5)$ & 8.87 & 8.87 & 0.28 & 0.25 & 0.27 & 0.51 & 0.44 & 0.47 & 2.239 & 2.069 \\
\hline $0.55(4.1)$ & 6.29 & 6.29 & 0.41 & 0.21 & 0.31 & 0.59 & 0.43 & 0.51 & 2.227 & 2.083 \\
\hline $0.733(5.3)$ & 1.45 & 1.78 & 0.89 & 0.16 & 0.53 & 1.23 & 0.32 & 0.78 & 2.191 & 2.091 \\
\hline
\end{tabular}



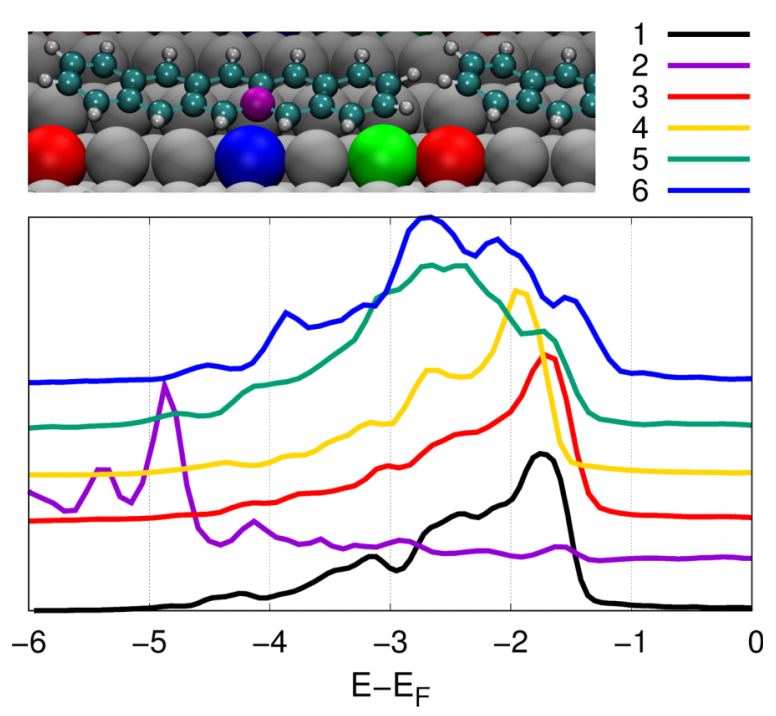

FIG. 9. Pn adsorption on the Fibonacci modulated copper surface. Local DOS for $\mathrm{C}$ and $\mathrm{Cu}$ atoms in the row structure. The Pn coverage is $0.733 \mathrm{ML}$ $\left(5.3 \times 10^{13}\right.$ molecules $\left.\mathrm{cm}^{-2}\right)$ for all cases except 1 and 4 . 1 : Local DOS for a surface $\mathrm{Cu}$ atom of the clean substrate. 2: Local DOS of a $\mathrm{C}$ atom in the middle part of the Pn molecule. 3: Local DOS for a surface $\mathrm{Cu}$ atom below the more bent end of the Pn molecule. 4: Local DOS for a surface $\mathrm{Cu}$ atom below the end of the Pn molecule at a coverage of $0.37 \mathrm{ML}\left(2.7 \times 10^{13}\right.$ molecules $\left.\mathrm{cm}^{-2}\right)$. 5: Local DOS for a surface $\mathrm{Cu}$ atom below the less bent end of the Pn molecule. 6: Local DOS for a surface $\mathrm{Cu}$ atom below the middle part of the Pn molecule. Inset: Key showing which atoms the curves correspond to. The yellow curve does not have an associated atom in this diagram because it is for a different coverage.

tween the ends of the molecules varies between molecules.

Figure 9 demonstrates the effect of bending on bonding of the molecule by local DOS plots for $\mathrm{C}$ and $\mathrm{Cu}$ atoms at different positions related to the molecule. This figure clearly shows that the bonding is strongest in the middle part of the Pn molecule. The bending weakens the bonding so that the higher the molecule end bends the weaker the bonding. However, the lower end of the bent molecules bonds more strongly to the surface than the end of unbent molecules in low coverage. This is because the distance between the lower end of the bended molecule and the substrate is smaller than the distance between the end of the unbent molecule and the substrate. On average, the bonding in bent molecules is stronger when the bending is asymmetric.
Figure 10 shows a three-dimensional plot of the partial charge density of a Pn molecule on the adsorption site 1 with coverage $0.733 \mathrm{ML}\left(5.3 \times 10^{13}\right.$ molecules $\left.\mathrm{cm}^{-2}\right)$. The colored energy regions ( -1 to $0 \mathrm{eV}$ yellow, -1.8 to $-1 \mathrm{eV}$ blue, -3.2 to $-1.8 \mathrm{eV} \tan ,-4.5$ to $-3.2 \mathrm{eV} \mathrm{red}$, and -5.2 to $-4.5 \mathrm{eV}$ green) correspond to the color coding in partial DOS in (a).

This clearly shows that the bonding is strongest at -4.5 to $-3.2 \mathrm{eV}$ (red). This is the energy region towards which the $\mathrm{Cu}$ DOS in Figure 9 spreads in the case of $\mathrm{Cu}$ atoms that are close to $\mathrm{C}$ atoms. Surprisingly the energy region -1 to $0 \mathrm{eV}$ (yellow) also shows weak bonding even if there is almost no charge for $\mathrm{Cu}$ or $\mathrm{C}$ in this region. Energy region -1.8 to -3.2 ( $\tan$ ) does not show bonding to the molecule, even if most of the charge for $\mathrm{Cu}$ remains in that region. Energy region -4.5 to -5.2 shows no bonding to the surface, although most of the $\mathrm{C}$ charge remains at that region.

Figure 10 also illustrates that the more bent end of the molecule does not bond to the surface. This same thing is illustrated in Figure 9 where the DOS for a $\mathrm{Cu}$ atom below the more bent end of the molecule remains similar to the DOS of clean surface $\mathrm{Cu}$, i.e., the curve 3 in Figure 9 is not spread towards the -4.5 to $-3.2 \mathrm{eV}$ (red) energy region.

At the chosen energy regions, we do not see any bonding between the molecules.

Table III lists adsorption energies as a function of the coverage. A more negative adsorption energy for a structure is associated with a greater preference for that structure. Column "row" shows the adsorption energies for one molecule, when only the best adsorption sites are occupied by Pn. Column "checkerboard" shows the adsorption energy for one molecule when the molecules are on the adsorption sites 1 and 5. The checkerboard energies are the averages of the adsorption energies of those two sites. For the coverages of $0.37 \mathrm{ML}\left(2.7 \times 10^{13}\right.$ molecules $\left.\mathrm{cm}^{-2}\right)$ and $0.34 \mathrm{ML}\left(2.4 \times 10^{13}\right.$ molecules $\left.\mathrm{cm}^{-2}\right)$, the distances between the molecules in the direction across $\mathrm{Cu}$ steps are $16.59 \AA$ and $19.15 \AA$. The distance between the molecules in the other direction is in both cases $7.42 \AA$. In this case, all distances are measured between the nearest $\mathrm{H}$ atoms. The identical adsorption energy indicates that these coverages are below the threshold for intermolecular interaction. Column "tilted row" shows the energy for the densest structure, achieved when molecules develop a tilt along their long axis, allowing them to decorate more rows. The "total adsorption
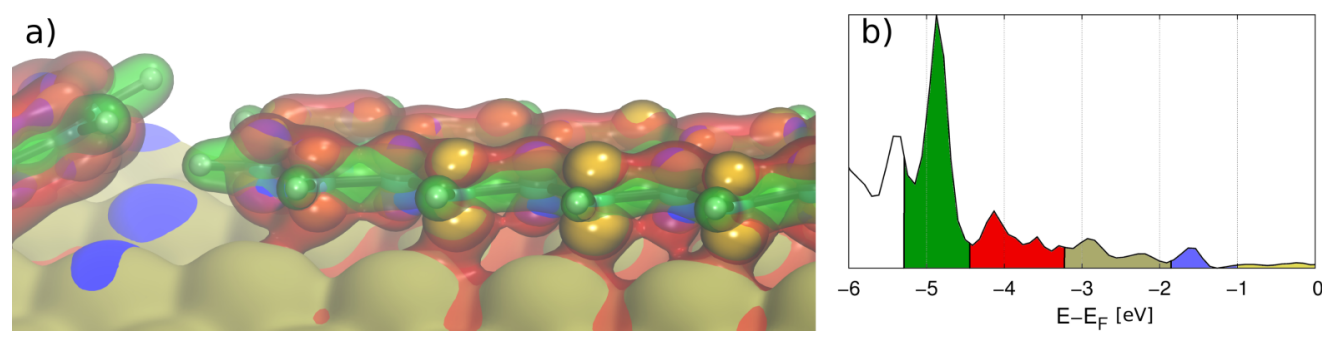

FIG. 10. Pn adsorption on the Fibonacci modulated copper surface. (a) 3-dimensional plot of the partial charge density of the Pn molecule on the adsorption site 1 with coverage $0.733 \mathrm{ML}\left(5.3 \times 10^{13}\right.$ molecules $\left.\mathrm{cm}^{-2}\right)$. (b) Local DOS of a C atom in the middle part of the Pn molecule (same curve as in Figure 9). The colored energy regions ( -1 to $0 \mathrm{eV}$ yellow, -1.8 to $-1 \mathrm{eV}$ blue, -3.2 to $-1.8 \mathrm{eV}$ tan, -4.5 to $-3.2 \mathrm{eV}$ red, and -5.2 to $-4.5 \mathrm{eV}$ green) correspond to the color coding in partial DOS in (a). 
TABLE III. Adsorption energies as a function of coverage.

\begin{tabular}{|c|c|c|c|c|}
\hline $\begin{array}{l}\text { Coverage (ML } \\
\left.\left(\cdot 10^{13} \text { molecules } \mathrm{cm}^{-2}\right)\right)\end{array}$ & $\begin{array}{c}\text { Adsorption energy } \\
\text { (eV) row }\end{array}$ & $\begin{array}{l}\text { Adsorption energy } \\
(\mathrm{eV}) \text { checkerboard }\end{array}$ & $\begin{array}{l}\text { Adsorption energy } \\
(\mathrm{eV}) \text { tilted row }\end{array}$ & $\begin{array}{l}\text { Total adsorption energy } \\
\qquad\left(\cdot 10^{13} \mathrm{eV} \mathrm{cm}^{-2}\right)\end{array}$ \\
\hline $0.34(2.4)$ & -4.75 & & & -11.4 \\
\hline $0.37(2.7)$ & -4.75 & & & -12.8 \\
\hline $0.40(2.9)$ & -4.74 & & & -13.7 \\
\hline $0.44(3.2)$ & -4.74 & & & -15.2 \\
\hline $0.49(3.5)$ & -4.73 & & & -16.6 \\
\hline $0.55(4)$ & -4.72 & -4.40 & & -18.8 to -17.6 \\
\hline $0.73(5.3)$ & -4.36 & -4.40 & & -23.1 to -23.3 \\
\hline $0.80(5.8)$ & & -4.32 & & -25.1 \\
\hline $0.88(6.4)$ & & -4.25 & & -27.2 \\
\hline $0.98(7.1)$ & & -4.06 & & -28.8 \\
\hline $1.10(8)$ & & -3.82 & & -30.4 \\
\hline $1.46(11)$ & & & -3.65 & -40.2 \\
\hline
\end{tabular}

energy" is the product of the number of molecules and each individual adsorption energy. This provides a useful indicator that the overall energy becomes more negative with increasing coverage throughout all the phases.

\section{CONCLUSIONS}

We have used DFT and STM to comprehensively investigate and model all stages of the growth of the first layer of pentacene molecules on a Fibonacci modulated $\mathrm{Cu}$ film. Molecules have a strong preference to decorate sites which allow them to orient their long axes parallel to the Fibonacci row structure in the $\mathrm{Cu}$ film. As coverage is increased, the molecules in the row structure get closer together, and the resulting steric effect causes enhanced bending on the molecules. Repulsion between the ends of the molecules forces a chain effect where one end of a molecule bends up and one end of a neighboring molecule bends down, as observed for $\mathrm{Cu}(111){ }^{32}$ This in turn weakens the moleculesurface interaction. The transition from the row structure to the checkerboard structure observed previously ${ }^{16}$ takes place at the critical coverage of $0.73 \mathrm{ML}\left(5.3 \times 10^{13}\right.$ molecules $\left.\mathrm{cm}^{-2}\right)$. After all of the film has transformed to the checkerboard structure, additional Pn molecules result in a further phase transition to a tilted row structure, observed in STM and DFT. These phase transitions are facilitated by the easy lengthwise sliding of molecules, evidenced both experimentally and using DFT.

\section{SUPPLEMENTARY MATERIAL}

See the supplementary material for the calculations of the adsorption site geometry of Pn adsorption perpendicular to the Fibonacci rows, for intermolecular distances in Pn adsorption site calculations and for XYZ-coordinates for the checkerboard structure with a coverage of $0.98 \mathrm{ML}$.

\section{ACKNOWLEDGMENTS}

The EPSRC is thanked for funding under Grant No. EP/D05253X/1 and Academy of Finland for funding under
Project Nos. 277829 and 218186. CSC_-IT Center for Science is thanked for the computational resources.

${ }^{1}$ J. A. Smerdon, K. M. Young, M. Lowe, S. S. Hars, T. P. Yadav, D. Hesp, V. R. Dhanak, A. P. Tsai, H. R. Sharma, and R. McGrath, Nano Lett. 14, 1184-1189 (2014)

${ }^{2}$ H. R. Sharma, K. Nozawa, J. A. Smerdon, P. J. Nugent, I. McLeod, V. R. Dhanak, M. Shimoda, Y. Ishii, A. P. Tsai, and R. McGrath, Nat. Commun. 4, 2715 (2013).

${ }^{3}$ J. Ledieu, J. T. Hoeft, D. E. Reid, J. A. Smerdon, R. D. Diehl, T. A. Lograsso, A. R. Ross, and R. McGrath, Phys. Rev. Lett. 92, 135507 (2004).

${ }^{4}$ K. Pussi, M. Gierer, and R. D. Diehl, J. Phys.: Condens. Matter 21, 474213 (2009).

${ }^{5}$ G. Bavdek, A. Cossaro, D. Cvetko, C. Africh, C. Blasetti, F. Esch, A. Morgante, and L. Floreano, Langmuir 24, 767-772 (2008).

${ }^{6}$ A. Baby, G. Fratesi, S. R. Vaidya, L. L. Patera, C. Africh, L. Floreano, and G. P. Brivio, J. Phys. Chem. C 119, 3624-3633 (2015).

${ }^{7}$ P. Schroeder, C. France, J. Park, and B. Parkinson, J. Appl. Phys. 91, 3010-3014 (2002).

${ }^{8}$ A. Ferretti, C. Baldacchini, A. Calzolari, R. Di Felice, A. Ruini, E. Molinari, and M. G. Betti, Phys. Rev. Lett. 99, 046802 (2007).

${ }^{9}$ X.-Q. Shi, Y. Li, M. A. Van Hove, and R.-Q. Zhang, J. Phys. Chem. C 116, 23603 (2012).

${ }^{10}$ K. Müller, A. Kara, T. K. Kim, R. Bertschinger, A. Scheybal, J. Osterwalder, and T. A. Jung, Phys. Rev. B 79, 245421 (2009).

${ }^{11}$ J. Lagoute, K. Kanisawa, and S. Fölsch, Phys. Rev. B 70, 245415 (2004).

${ }^{12}$ N. Koch, A. Gerlach, S. Duhm, H. Glowatzki, G. Heimel, A. Vollmer, Y. Sakamoto, T. Suzuki, J. Zegenhagen, J. P. Rabe, and F. Schriber, J. Am. Chem. Soc. 130, 7300 (2008).

${ }^{13}$ V. Ignatescu, J.-C. M. Hsu, A. C. Mayer, J. M. Blakely, and G. G. Malliaras, Appl. Phys. Lett. 89(25), 253116 (2006).

${ }^{14}$ C. Baldacchini, M. G. Betti, V. Corradini, and C. Mariani, Surf. Sci. 566, 613-617 (2004).

${ }^{15}$ L. Gavioli, M. Fanetti, D. Pasca, M. Padovani, M. Sancrotti, and M. G. Betti, Surf. Sci. 566, 624-627 (2004).

${ }^{16}$ K. M. Young, J. A. Smerdon, H. R. Sharma, M. Lahti, K. Pussi, and R. McGrath, Phys. Rev. B 87, 085407 (2013).

${ }^{17}$ I. R. Fisher, M. J. Kramer, T. A. Wiener, Z. Islam, A. R. Ross, T. A. Lograsso, A. Kracher, A. I. Goldman, and P. C. Canfield, Philos. Mag. B 79(10), 1673-1684 (1999).

${ }^{18}$ G. Kresse and J. Hafner, Phys. Rev. B 47, 558 (1993).

${ }^{19}$ G. Kresse and J. Hafner, J. Phys.: Condens. Matter 6, 8245 (1994).

${ }^{20} \mathrm{G}$. Kresse and J. Hafner, Phys. Rev. B 49, 14251 (1994).

${ }^{21}$ G. Kresse and J. Furthmüller, Comput. Mater. Sci. 6, 15 (1996).

${ }^{22}$ G. Kresse and J. Furthmüller, Phys. Rev. B 54, 11169 (1996).

${ }^{23}$ P. E. Blöchl, Phys. Rev. B 50, 17953 (1994).

${ }^{24}$ J. P. Perdew, K. Burke, and M. Ernzerhof, Phys. Rev. Lett. 77, 3865 (1996).

${ }^{25}$ H. J. Monkhorst and J. D. Pack, Phys. Rev. B 13, 5188 (1976).

${ }^{26}$ J. Klimeš, D. R. Bowler, and A. Michaelides, Phys. Rev. B 83, 195131 (2011).

${ }^{27}$ H. Yildirim, T. Greber, and A. Kara, J. Phys. Chem. C 117, 20572 (2013). 
${ }^{28}$ J. Carrasco, W. Liu, A. Michaelides, and A. Tkatchenko, J. Chem. Phys. 140, 084704 (2014).

${ }^{29}$ K. Müller, A. P. Seitsonen, T. Brugger, J. Westover, T. Greber, T. Jung, and A. Kara, J. Phys. Chem. C 116(44), 23465-23471 (2012).

${ }^{30}$ E. Annese, C. E. Viol, B. Zhou, J. Fujii, I. Vobornik, C. Baldacchini, M. G. Betti, and G. Rossi, Surf. Sci. 601, 4242 (2007).
${ }^{31}$ S. Lukas, G. Witte, and C. Wöll, Phys. Rev. Lett. 88, 028301 (2001).

${ }^{32}$ J. A. Smerdon, M. Bode, N. P. Guisinger, and J. R. Guest, Phys. Rev. B 84, 165436 (2011).

${ }^{33}$ L. Gross, F. Mohn, N. Moll, P. Liljeroth, and G. Meyer, Science 325, 1110 (2009). 